\title{
Evaluation of a revised resuscitation protocol for out-of-hospital cardiac arrest patients due to COVID- 19 safety protocols: A single-center retrospective study in Japan
}

Kenji Kandori ( $\square$ knj.kandori@gmail.com )

Japanese Red Cross Society Kyoto Daini Hospital

Yohei Okada

Preventive Services, School of Public Health, Kyoto University

Wataru Ishii

Japanese Red Cross Society Kyoto Daini Hospital

Hiromichi Narumiya

Japanese Red Cross Society Kyoto Daini Hospital

Ryoji lizuka

Japanese Red Cross Society Kyoto Daini Hospital

\section{Research Article}

Keywords: cardiopulmonary resuscitation, COVID-19, infection control, out-of-hospital cardiac arrest, personal protective equipment, resuscitation protocol

Posted Date: March 16th, 2021

DOl: https://doi.org/10.21203/rs.3.rs-289836/v1

License: (c) (1) This work is licensed under a Creative Commons Attribution 4.0 International License.

Read Full License 


\section{Abstract}

Background: This study aimed to determine the association between cardiopulmonary resuscitation (CPR) under the coronavirus 2019 (COVID-19) safety protocols in our hospital and the prognosis of outof-hospital cardiac arrest (OHCA) patients, in an urban area tertiary critical care center, where the prevalence of COVID-19 infection is relatively low.

Methods: This was a single-center, retrospective, observational, cohort study conducted at a tertiary critical care center in Kyoto City, Japan. Adult OHCA patients were included who were admitted to our emergency department while still in cardiac arrest at hospital arrival between January 1, 2019, and December 31, 2020. Our hospital implemented a revised resuscitation protocol for OHCA patients on April 1, 2020 to prevent COVID-19 disease transmission. This study defined the conventional CPR period as January 1, 2019 to March 31, 2020, and the COVID-19 safety protocol period as April 1, 2020 to December 31,2020 . The primary outcome was hospitalization survival. The secondary outcomes were return of spontaneous circulation after hospital admission and 1-month survival after OHCA occurrence. The adjusted odds ratios with $95 \%$ confidence intervals $(\mathrm{Cl})$ were calculated for outcomes to compare the two study periods, and the multivariable logistic model was used to adjust for six variables: age, presence of witness, presence of bystander CPR, initial cardiac rhythm at the scene, call-hospital interval, and the first documented cardiac rhythm at hospital arrival.

Results: The study analyzed 443 patients, with a median age of 76 years (65-85), and included 261 men (58.9\%). The percentage of hospitalization survivors during the entire research period was $16.9 \%$ (75/443 patients), with 18.7\% (50/267) during the conventional CPR period and 14.2\% (25/176) during the COVID19 safety protocol period. The adjusted odds ratio for hospitalization survival during the COVID-19 safety protocol period was $0.61(95 \% \mathrm{Cl}, 0.32-1.18)$, as compared with conventional CPR.

Conclusions: There was no apparent difference in hospitalization survival between the OHCA patients resuscitated under the conventional CPR protocol compared with the current revised protocol for controlling COVID-19 disease transmission.

\section{Background}

The incidence of coronavirus disease (COVID-19) continues to increase worldwide. To prevent further spreading, the American Heart Association and the European Resuscitation Council have published revised guidelines for resuscitation of out-of-hospital cardiac arrest (OHCA) patients [1, 2]. During resuscitation, aerosols are potentially generated and health care providers should take precautions against COVID-19 transmission in prehospital and hospital settings. In the guidelines, wearing personal protective equipment (PPE) with an N-95 mask is especially recommended. However, there are potential disadvantages, including communication difficulties between team members and a decreased quality of the resuscitation such as chest compression procedures [3-8]. Even though the safety of health care provider is the first priority, the prognosis of OHCA patients is also an important issue. On April 1, 2020, a 
revised resuscitation protocol was implemented in our hospital to prevent spreading of COVID-19; however, it is unclear whether the revised protocol has affected the patients' prognoses. If the quality of resuscitation has been compromised by the COVID-19 safety protocols and patient outcomes made worse, the protocols should be improved as much as possible. However, there are few studies that investigate the association between the precautions for COVID-19 and the outcomes of OHCA patients in areas where the prevalence of COVID-19 is relatively low [9-11].

The aim of this study was to evaluate the association between in-hospital resuscitation under COVID-19 safety protocols, in an urban area tertiary critical care center where the prevalence of COVID-19 infection is relatively low, and the prognoses of OHCA patients.

\section{Methods}

\section{Study design}

This is a single-center, retrospective, observational, cohort study. The study was approved by the Clinical Research Ethics Committee of the Japanese Red Cross Society Kyoto Daini Hospital (Approval ID Sp2020-11). The Ethics Committee waived the requirement for informed consent because of the anonymous nature of the data. All procedures in this study were performed in accordance with relevant guidelines and regulations.

\section{Setting}

This study was performed at a tertiary critical care center in Kyoto City, Japan, which is an urban area with a population of approximately 1.5 million and about 90,000 ambulance calls annually [12]. Our 672bed hospital is one of four tertiary critical care medical centers in Kyoto City. Generally, tertiary critical care medical centers in Japan can accept emergency and critically ill patients transported by ambulance, including sepsis, acute coronary syndrome, cardiac arrest, severe trauma, and stroke patients, and can provide specialized treatment in an intensive care unit [13]. In 2019, the emergency department cases had 7,610 patients who arrived by ambulance and 20,769 patients of "walk-in" status that arrived by other means [13].

\section{Study population}

This study included adult patients (age $\geq 18$ years) with OHCA who were admitted to our emergency department while still in cardiac arrest at hospital arrival between January 1,2019, and December 31, 2020. This study excluded OHCA patients who had a return of spontaneous circulation (ROSC) at hospital arrival.

\section{COVID-19 cases in Kyoto City}

In Kyoto City, the first case of COVID-19 infection was confirmed on January 30, 2020; by the end of March, 42 cases had been confirmed. After that, the number of infections increased through mid-May; by 
the end of May there were 248 cases. The number of infections decreased briefly; however, since late June, the number of cases continued to increase bimodally and reached 3,369 by December 31, 2020 [14] (Supplementary Figure 1).

\section{Emergency medical service resuscitation protocol in Kyoto City}

During the COVID-19 infection-spreading period, the emergency medical service (EMS) in Kyoto city implemented a protocol to treat all the cardiac arrest patients as possibly having COVID-19. Bag-valve mask (BVM) ventilation and chest compression were performed with attention to the fact that viruscontaining aerosols might be generated. In the EMS protocol, before entering the scene, all staff donned PPE that included N95 masks and eye protection, and a high-efficiency particulate air (HEPA) filter was attached securely to any manual or mechanical ventilation device in the path of exhaled gas. Breathing was assessed by observing chest wall movement in order to minimize the risk of infection. Chest compression was started after covering the mouth and nose of the patient with a BVM and holding it close to the patient's face. Chest compression was limited to as short a time as possible when the mouth of the patient was not covered with a mask or when advanced airway management such as laryngeal tube or tracheal intubation was not introduced. It was recommended that advanced airway management be introduced as early as possible. When administering positive pressure ventilation with a BVM, EMS staff held the BVM tightly against the patient's face to minimize air leakage. Since EMS personnel are generally not allowed to terminate resuscitation in a prehospital setting, all OHCA cases were transported to a hospital.

\section{Revised resuscitation protocol during the COVID-19 period in our hospital}

In the interest of controlling COVID-19 transmission, our hospital implemented a revised resuscitation protocol for OHCA patients on April 1, 2020. The details are described in the additional file. In brief, a restricted zone separated from other emergency beds by doors or plastic curtains was set up during resuscitation, and OHCA patients were admitted and treated only in this space (Supplementary Figure 2). All staff involved in the resuscitation procedures were required to wear PPE, including N95 masks (Supplementary Figure 3). An attending emergency physician was placed outside the isolated resuscitation area to direct the other team members who performed the resuscitation activities. The conventional cardiopulmonary resuscitation (CPR) period was defined as January 1, 2019 to March 31, 2020, and the COVID-19 safety protocol period was April 1, 2020 to December 31, 2020.

\section{Data collection}

Prehospital resuscitation data and in-hospital data were obtained by electronic chart reviews by certified emergency physician. Prehospital resuscitation data included the presence of a witness, presence of a bystander who performed CPR, initial cardiac rhythm at the scene, prehospital epinephrine administration, prehospital advanced airway management, prehospital automated external defibrillator use, the callhospital interval, and achievement of prehospital ROSC. The call-hospital interval was defined as the period from the incoming call to the time when the patient arrived at the hospital. In-hospital data 
included baseline characteristics of the patients (age and sex), treatments such as coronary angiography, and use of a mechanical circulatory device (extracorporeal membrane oxygen and/or intra-aortic balloon pumping). The cause of arrest was defined as having a cardiac (e.g., acute coronary syndrome, other heart disease, presumed cardiac cause), non-cardiac (e.g., cerebrovascular diseases, respiratory diseases, malignant tumors), or an external cause (including traffic injury, fall, hanging, drowning, asphyxia, drug overdose, or any other external cause) $[15,16]$. The medical cause was defined as the cause of arrest other than an external cause. Patients were categorized by age as $18-64$ years, $65-74$ years, and $\geq 75$ years. Patient outcomes were also collected.

\section{Outcome measures}

The primary outcome of the study was survival of hospitalization. The secondary outcomes were ROSC after hospital admission and 1-month survival after OHCA occurrence.

\section{Selection of variables}

Based on previous studies [16-20], six potential confounding factors were selected: age, presence of a witness, presence of bystander CPR, initial cardiac rhythm at the scene, the call-hospital interval, and the first documented cardiac rhythm at hospital arrival.

\section{Sample size estimation}

It was estimated that at least $60-70$ case outcomes would be required to account for the confounders using a logistic model, based on the generally accepted rule of 10 events per variable [21]. Considering this, it was determined that including cases from January 2019 to March 2020, before implementation of the COVID-19 safety protocols, would result in an adequate sample size for analysis.

\section{Statistical analysis}

Data statistics for patient characteristics were calculated as a median with an interquartile range (IQR) for continuous variables and as a number with percent for categorical variables. The crude and adjusted odds ratios (AORs) of outcomes with 95\% confidence intervals (Cls) were calculated using the multivariable logistic model including all potential confounders. Missing data were not replaced or estimated. Statistical analyses were performed using JMP Pro 14 software (SAS Institute, Cary, NC, USA).

\section{Results}

\section{Patient characteristics}

Of the 484 OHCA patients admitted to our emergency department between January 1,2019, and December 31, 2020, 7 patients aged $<18$ years were excluded. Another 34 patients were excluded because they obtained ROSC at hospital arrival. The remaining 443 patients were included in the analysis (Fig. 1). Patient characteristics are shown in Table 1. In summary, the median age was 76 years (IQR, 65-85), and 
261 (58.9\%) patients were men. The baseline characteristics were similar between the conventional and COVID-19 safety protocol period. Further, during the study period, there was no confirmed case of infected medical staff involved in a resuscitation in our hospital.

\section{Outcomes}

The primary and secondary outcomes are shown in Table 2. As primary outcome, the percentage of hospitalization survivors in the entire research period was $16.9 \%$ (75/443 patients). During the conventional CPR period (January 2019-March 2020), it was 18.7\% (50/267) compared with $14.2 \%$ (25/176) for the COVID-19 safety protocol period (April-December 2020).

As secondary outcome, the incidence of ROSC after hospital admission during the entire research period was $34.3 \%$ (152/443 patients); during the conventional CPR period, it was 33.3\% (89/267), as compared with $35.8 \%$ (63/176) for the COVID-19 infection safety protocol period. The rate of 1-month survival after OHCA occurrence during the entire research period was 5.2\% (23/443 patients); during the conventional CPR period, it was $5.2 \%$ (14/267), as compared with $5.1 \%$ (9/176) for the COVID-19 safety protocol period.

\section{Primary analysis}

For the primary analysis, multivariable logistic regression analysis showed that the AOR for hospitalization survival during the COVID-19 safety protocol period was 0.61 ( $95 \% \mathrm{Cl}, 0.32-1.18$ ), as compared with conventional CPR (Table 2). The AORs of other confounders are shown in Supplementary Table 1.

For the secondary outcomes, multivariable logistic regression analysis showed that the AOR for ROSC after hospital admission during the COVID-19 safety protocol period was 1.11 ( $95 \% \mathrm{Cl}, 0.69-1.79$ ), as compared with conventional CPR (Table 2). For 1-month survival, the AOR of the COVID-19 safety protocol period was 1.14 (95\% Cl, 0.37-3.50), as compared with conventional CPR (Table 2).

\section{Discussion}

\section{Key observations}

The study results suggest that there was no difference in the survival outcomes between OHCA patients resuscitated under the current COVID-19 safety protocol compared with those treated before the protocol was implemented. This suggests that CPR under the current infection control measures for COVID-19 is be able to ensure the quality of resuscitation, and is acceptable for continuance. Furthermore, there were no cases of infection among the staff involved in the resuscitation in our hospital, suggesting that their safety is also accounted for.

\section{Strengths Of The Study}


One strength of this study is its setting in a non-pandemic area. The majority of previous studies on OHCA were conducted in COVID-19 pandemic areas [22-34]. Most of these studies reported that OHCA resuscitation attempted at the scene declined during the COVID-19 pandemic [22-24, 26-32]. Therefore, there is a limitation to applying the results of studies in pandemic regions to non-pandemic regions. On the other hand, some of the regions have not reached, or are recovering from, a COVID-19 pandemic. For such a non-pandemic setting, the result in this study may be valuable.

Second, this is the first study to focus on patient outcomes before and after the in-hospital resuscitation protocol changes due to COVID-19 safety protocols among OHCA patients. Most previous studies focused on prehospital settings such as arrest witnesses and bystander CPR [9-11, 22-31, 33]. However, in-hospital resuscitation has been also changing due to COVID-19 safety protocols, which may limit the number of personnel involved in resuscitation, make communication more difficult, and increase the physical and psychological burden compared to conventional resuscitation $[1-3,8]$. A previous study indicated that percutaneous coronary intervention was withheld even after transport to the hospital [34]. Therefore, it was hypothesized that changes in in-hospital resuscitation might potentially result in poor prognoses of OHCA patients, and it is important to examine the association between the prognoses of OHCA patients and in-hospital resuscitation under COVID-19 safety protocols. However, the study result did not support the hypothesis. Because resuscitation under COVID-19 safety protocols is expected to continue in the future, this result may be helpful when considering in-hospital resuscitation strategies.

The third strength of this study is that the results may be valid due to the design being considered a kind of natural experiment. A natural experiment is defined as a way to assess the effect of interventions or policy changes for which planned and controlled experimental research designs may be infeasible or inappropriate to implement $[13,35,36]$. Similar to a randomized controlled trial, the approach to this study has a strength in that the patient's background and treatment can be considered as equipoise before and after the intervention, and that the effects of unmeasured confounding may be less

pronounced. Therefore, this type of study design has attracted interest as an alternative to a randomized controlled trial [36]. Since a randomized trial on resuscitation under COVID-19 safety protocols is neither practical nor ethically feasible to conduct, this current study, the effect of COVID-19 safety protocols on resuscitation could be evaluated as if it was an experiment, albeit not under control. Therefore, since the study area was considered to be less affected by COVID-19 infection, and the patient background after the revised protocol was almost same as in before, then the presence or absence of the COVID-19 safety protocol can be considered the only variable in the two cohorts, and the effect of unmeasured confounding is likely to be small. In this regard, the results of this study should be highly valid.

\section{Interpretation Of The Results}

The results of this study suggest that resuscitation under COVID-19 safety protocols do not strongly affect the prognoses of OHCA patients. There are some possible reasons for this. The first is the proficiency of resuscitation wearing PPE. In our emergency room, health care providers wear the PPE not only in resuscitation but also when treating patients with possible COVID-19 infection such as those with 
fever. In addition, when performing tracheal intubation, the procedure is always performed under PPE that includes $\mathrm{N} 95$ masks. Therefore, health care providers became accustomed to performing medical treatments and procedures under the COVID-19 safety protocols, which may help retain the quality of resuscitation.

Secondly, in all cases, attending emergency physicians are placed outside the restricted zone and resuscitation is performed under their supervision; this could minimize the confusion at the resuscitation area and sustain the quality of CPR. Generally, the actual scene of resuscitation is sometimes chaotic under normal conditions. Further, the difficulty in communicating among the members due to the wearing of PPE, including N95 masks, may lead to losing the necessary command and control. In addition, even if the medical staff is familiar with PPE, decision-making for the resuscitation strategy in a stressful situation wearing PPE might cause a high degree of physical and mental fatigue. This might threaten not only the quality of resuscitation but also the safety of the staff. In our hospital, the attending emergency physician directs the resuscitation and makes decisions outside the isolation room while keeping an eye on the safety of the staff performing the CPR. This enables the team members to focus on the procedures while attending to their own safety. This suggests that it is possible to guarantee the quality of resuscitation while ensuring safety.

\section{Limitations}

This study has several limitations. First, the sample size was limited and the statistical power might be inadequate to detect differences in the outcomes. Even though this limitation is understood, the study was necessary because if resuscitation under the current safety protocol had led to significantly worse poor outcomes, immediate improvements would have been needed. Further verification with a larger sample size is needed. Second, although this study is like as a natural experiment, some potential unmeasured confounders might influence the results. Third, regarding the single-center study design, the generalizability of these results to another hospital is unclear. Fourth, the results may also vary in our hospital if the study period is different. If the number of COVID-19 cases increases dramatically in the future, the prognoses may be poor, as in reported pandemic areas. Furthermore, because a polymerase chain reaction test was not performed for all OHCA patients, the number of OHCA patients with COVID-19 included in this study was unknown.

\section{Conclusions}

This study showed that there is no significant change in hospitalization survival outcomes between OHCA patients treated by conventional CPR and those treated under the current measures for controlling COVID-19 transmission.

\section{List Of Abbreviations}

AOR, adjusted odds ratio 
BVM, bag-valve mask

$\mathrm{Cl}$, confidence interval

COVID-19, coronavirus disease

CPR, cardiopulmonary resuscitation

EMS, emergency medical service

HEPA, high-efficiency particulate air

IQR, interquartile range

OHCA, out-of-hospital cardiac arrest

PPE, personal protective equipment

ROSC, return of spontaneous circulation

\section{Declarations}

\section{Ethics approval and consent to participate}

The Ethics Committee of Japanese Red Cross Society Kyoto Daini Hospital approved this study protocol (Sp2020-11), and the requirement of written informed consent was waived.

\section{Consent for publication}

Not applicable

\section{Availability of data and materials}

Not applicable

\section{Competing interests}

The authors declare that they have no competing interests.

\section{Funding}

Not applicable

\section{Authors' contributions}

KK contributed to the conception and design of this work, formal analysis, and writing the original draft. YO provided statistical advice on the study design and analyzed the data. WI, HN, and RI supervised the 
study. All authors read and approved the final manuscript.

\section{Acknowledgments}

We appreciate all the staff of the Japanese Red Cross Society Kyoto Daini Hospital for their contribution.

\section{References}

1. Edelson, D. P. et al. Interim guidance for basic and advanced life support in adults, children, and neonates with suspected or confirmed COVID-19: From the Emergency Cardiovascular Care Committee and Get With The Guidelines-Resuscitation Adult and Pediatric Task Forces of the American Heart Association. Circulation.141, e933-e43 (2020).

2. Nolan, J. P. et al. European Resuscitation Council COVID-19 guidelines executive summary. Resuscitation.153, 45-55 (2020).

3. Kandori, K., Narumiya, H. \& lizuka, R. Extracorporeal cardiopulmonary resuscitation should not be performed on confirmed or suspected COVID-19 patients. Resuscitation.153, 6-7 (2020).

4. Sahu, A. K., Suresh, S., Mathew, R., Aggarwal, P. \& Nayer, J. Impact of personal protective equipment on the effectiveness of chest compression - A systematic review and meta-analysis. Am. J. Emerg. Med.39, 190-196 (2021).

5. Małysz, M. et al. An optimal chest compression technique using personal protective equipment during resuscitation in the COVID-19 pandemic: a randomized crossover simulation study. Kardiol. Pol.78, 1254-1261 (2020).

6. Malysz, M. et al. Resuscitation of the patient with suspected/confirmed COVID-19 when wearing personal protective equipment: A randomized multicenter crossover simulation trial. Cardiol. J.27, 497-506 (2020).

7. Smereka, J., Szarpak, L., Filipiak, K. J., Jaguszewski, M. \& Ladny, J. R. Which intravascular access should we use in patients with suspected/confirmed COVID-19? Resuscitation.151, 8-9 (2020).

8. Chen, J., Lu, K. Z., Yi, B. \& Chen, Y. Chest compression with personal protective equipment during cardiopulmonary resuscitation: A randomized crossover simulation study. Med. (Baltim).95, e3262 (2016).

9. Chan, P. S. et al. Outcomes for out-of-hospital cardiac arrest in the United States during the coronavirus disease 2019 pandemic.JAMA Cardiol. 2020.

10. Elmer, J., Okubo, M., Guyette, F. X. \& Martin-Gill, C. Indirect effects of COVID-19 on OHCA in a low prevalence region. Resuscitation.156, 282-283 (2020).

11. Uy-Evanado, A. et al. Out-of-hospital cardiac arrest response and outcomes during the COVID-19 pandemic. JACC Clin. Electrophysiol.7, 6-11 (2021).

12. Department, K. C. F. Kyoto City Fire Department: Emergency statistics. https://www.city.kyoto.lg.jp/shobo/page/0000279510.html Accessed 22 Feb 2021. 
13. Kandori, K. et al. Association between visitation restriction during the COVID-19 pandemic and delirium incidence among emergency admission patients: A single-center retrospective observational cohort study in Japan. J. Intensive Care.8, 1-9 (2020).

14. Kyoto City. The number of infected patients. https://www.city.kyoto.lg.jp/shobo/page/0000279510.html Accessed 22 Feb 2021.

15. Kitamura, T. et al. The profile of Japanese Association for Acute Medicine - out-of-hospital cardiac arrest registry in 2014-2015. Acute Med. Surg.5, 249-258 (2018).

16. Kandori, K. et al. Association between cardiac rhythm conversion and neurological outcome among cardiac arrest patients with initial shockable rhythm: A nationwide prospective study in Japan. Eur. Heart J. Acute Cardiovasc. Care.14, zuaa018 Online ahead of print. (2020 Oct).

17. Daya, M. R. et al. Out-of-hospital cardiac arrest survival improving over time: Results from the Resuscitation Outcomes Consortium (ROC). Resuscitation.91, 108-115 (2015).

18. Cournoyer, A. et al. Prognostic impact of the conversion to a shockable rhythm from a non-shockable rhythm for patients suffering from out-of-hospital cardiac arrest. Resuscitation.140, 43-49 (2019).

19. Cournoyer, A. et al. Impact of the direct transfer to percutaneous coronary intervention-capable hospitals on survival to hospital discharge for patients with out-of-hospital cardiac arrest. Resuscitation.125, 28-33 (2018).

20. Kragholm, K. et al. Return to work in out-of-hospital cardiac arrest survivors: A nationwide registerbased follow-up study. Circulation.131, 1682-1690 (2015).

21. Peduzzi, P., Concato, J., Kemper, E., Holford, T. R. \& Feinstein, A. R. A simulation study of the number of events per variable in logistic regression analysis. J. Clin. Epidemiol.49, 1373-1379 (1996).

22. Baldi, E. et al. Out-of-hospital cardiac arrest during the Covid-19 outbreak in Italy. N. Engl. J. Med.383, 496-498 (2020).

23. Baldi, E. et al. COVID-19 kills at home: The close relationship between the epidemic and the increase of out-of-hospital cardiac arrests. Eur. Heart. J.41, 3045-3054 (2020).

24. Baldi, E. et al. Treatment of out-of-hospital cardiac arrest in the COVID-19 era: A 100 days experience from the Lombardy region. PLoS One.15, e0241028 (2020).

25. Semeraro, F. et al. Out-of-hospital cardiac arrest during the COVID-19 era in Bologna: System response to preserve performances. Resuscitation.157, 1-2 (2020).

26. Paoli, A., Brischigliaro, L., Scquizzato, T., Favaretto, A. \& Spagna, A. Out-of-hospital cardiac arrest during the COVID-19 pandemic in the Province of Padua, Northeast Italy. Resuscitation.154, 47-49 (2020).

27. Lai, P. H. et al. Characteristics associated with out-of-hospital cardiac arrests and resuscitations during the novel coronavirus disease 2019 pandemic in New York City.JAMA Cardiol. 2020.

28. Fothergill, R. T., Smith, A. L., Wrigley, F. \& Perkins, G. D. Out-of-hospital cardiac arrest in London during the COVID-19 pandemic. Resusc. Plus.5, 100066 (2021). 
29. Marijon, E. et al. Out-of-hospital cardiac arrest during the COVID-19 pandemic in Paris, France: A population-based, observational study. Lancet Public Health.5, e437-e43 (2020).

30. Baert, V. et al. Assessment of changes in cardiopulmonary resuscitation practices and outcomes on 1005 victims of out-of-hospital cardiac arrest during the COVID-19 outbreak: Registry-based study. Scand J Trauma Resusc Emerg. Med.28, 119 (2020).

31. Rosell Ortiz, F. et al. Influence of the Covid-19 pandemic on out-of-hospital cardiac arrest. A Spanish nationwide prospective cohort study. Resuscitation.157, 230-240 (2020).

32. Yang, F., Yuan, Y., Song, Y. \& Lu, X. The effect on the out-of-hospital system of patients with out-ofhospital cardiac arrest during the COVID-19 outbreak in one city in China. Ann. Emerg. Med.76,687689 (2020).

33. Ball, J. et al. Collateral damage: Hidden impact of the COVID-19 pandemic on the out-of-hospital cardiac arrest system-of-care. Resuscitation.156, 157-163 (2020).

34. Rashid Hons, M. et al. Impact of coronavirus disease 2019 pandemic on the incidence and management of out-of-hospital cardiac arrest in patients presenting with acute myocardial infarction in England. J. Am. Heart Assoc.9, e018379 (2020).

35. Craig, P. et al. Using natural experiments to evaluate population health interventions: new Medical Research Council guidance. J. Epidemiol. Community Health.66, 1182-1186 (2012).

36. Craig, P., Katikireddi, S. V., Leyland, A. \& Popham, F. Natural experiments: An overview of methods, approaches, and contributions to public health intervention research. Annu. Rev. Public Health.38, 39-56 (2017).

\section{Tables}

Table 1. Patient characteristics between the conventional CPR and COVID-19 safety protocol period 
Variables, number, (\% or IQR)

All
patients
$(\mathrm{N}=443)$

Age, years

Age group, $n$

$76[65-$

85]
The conventional CPR period

(2019.01-2020.03)

$(\mathrm{N}=267)$
The COVID-19 safety protocol period (2020.04-2020.12)

$(\mathrm{N}=176)$

\begin{tabular}{|c|c|c|c|}
\hline 18-64 years & $\begin{array}{c}110 \\
(24.8 \%)\end{array}$ & $65(24.3 \%)$ & $45(25.6 \%)$ \\
\hline $65-74$ years & $\begin{array}{c}87 \\
(19.6 \%)\end{array}$ & $51(19.1 \%)$ & $36(20.5 \%)$ \\
\hline 75 years $\leq$ & $\begin{array}{c}246 \\
(55.5 \%)\end{array}$ & $151(56.6 \%)$ & $95(54.0 \%)$ \\
\hline Sex, Men, $\mathbf{n}$ & $\begin{array}{c}261 \\
(58.9 \%)\end{array}$ & $164(61.4 \%)$ & $97(55.1 \%)$ \\
\hline \multicolumn{4}{|l|}{ Cause of cardiac arrest, $n$} \\
\hline Cardiac cause & $\begin{array}{c}236 \\
(53.3 \%)\end{array}$ & $141(52.8 \%)$ & $95(54 \%)$ \\
\hline Cerebrovascular cause & $9(2.0 \%)$ & $8(3.0 \%)$ & $1(0.6 \%)$ \\
\hline Respiratory cause & $\begin{array}{c}24 \\
(5.4 \%)\end{array}$ & $12(4.5 \%)$ & $12(6.8 \%)$ \\
\hline Malignant tumor & $\begin{array}{c}14 \\
(3.2 \%)\end{array}$ & $9(3.4 \%)$ & $5(2.8 \%)$ \\
\hline External cause & $\begin{array}{c}135 \\
(30.5 \%)\end{array}$ & $80(30.0 \%)$ & $55(31.3 \%)$ \\
\hline Others or unknown & $\begin{array}{c}25 \\
(5.6 \%)\end{array}$ & $17(6.4 \%)$ & $8(4.5 \%)$ \\
\hline \multicolumn{4}{|l|}{ Pre-hospital information } \\
\hline Witnessed arrest, $\mathbf{n}$ & $\begin{array}{c}160 \\
(36.1 \%)\end{array}$ & $95(35.6 \%)$ & $65(36.9 \%)$ \\
\hline Bystander CPR, $n$ & $\begin{array}{c}160 \\
(36.1 \%)\end{array}$ & $94(35.2 \%)$ & $66(37.5 \%)$ \\
\hline \multicolumn{4}{|l|}{$\begin{array}{l}\text { Initial cardiac rhythm at the } \\
\text { scene }\end{array}$} \\
\hline Shockable rhythm & $\begin{array}{c}35 \\
(7.9 \%)\end{array}$ & $17(6.4 \%)$ & $18(10.2 \%)$ \\
\hline PEA & $\begin{array}{c}130 \\
(29.3 \%)\end{array}$ & $85(31.8 \%)$ & $45(25.6 \%)$ \\
\hline Asystole & $\begin{array}{c}264 \\
(59.6 \%)\end{array}$ & $155(58.1 \%)$ & $109(61.9 \%)$ \\
\hline $\begin{array}{l}\text { Pre-hospital epinephrine } \\
\text { administration, } n\end{array}$ & $\begin{array}{c}73 \\
(16.5 \%)\end{array}$ & $53(19.9 \%)$ & $20(11.4 \%)$ \\
\hline $\begin{array}{l}\text { Pre-hospital advanced airway } \\
\text { management, } n\end{array}$ & $\begin{array}{c}215 \\
(48.5 \%)\end{array}$ & $127(47.6 \%)$ & $88(50.0 \%)$ \\
\hline Call-hospital interval, min & $\begin{array}{c}28[23- \\
34]\end{array}$ & $28[22-33]$ & $29[25-34]$ \\
\hline
\end{tabular}

\section{In-hospital information}

77 [65-85]

76 [64-84]

\section{4]}

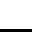




\begin{tabular}{cccc}
\hline Tracheal intubation, $\mathbf{n}$ & 366 & $220(82.4 \%)$ & $146(83 \%)$ \\
\hline Intervention, $\mathbf{n}$ & $(82.6 \%)$ & & \\
\hline VA-ECMO & & & $10(5.7 \%)$ \\
& 25 & $15(5.6 \%)$ & $5(2.8 \%)$ \\
\hline IABP & $(5.6 \%)$ & $12(4.5 \%)$ & $7(4.0 \%)$ \\
& $17 \%(1.7 \%)$ & $3(10)$ \\
\hline CAG & 23 & $16(6.0 \%)$ & $10(5.7 \%)$ \\
\hline PCI & $(5.2 \%)$ & $8(3.0 \%)$ & \\
\hline
\end{tabular}

Values are median (interquartile range [IQR]) or number (percentage). CAG, coronary angiography; COVID-19, coronavirus 2019; CPR, cardiopulmonary resuscitation; IABP, intra-aortic balloon pumping; IQR, interquartile range; OHCA, out-of-hospital cardiac arrest; PCI, percutaneous coronary intervention; PEA, pulseless electrical activity; ROSC, return of spontaneous circulation; TTM, targeted temperature management; VA-ECMO, veno-arterial extracorporeal membrane oxygenation

Table 2. The survival outcomes and multivariable logistic regression analysis for outcomes during the COVID-19 safety protocol period

\begin{tabular}{|c|c|c|c|c|c|}
\hline \multirow{2}{*}{$\begin{array}{l}\text { Outcomes, number, } \\
\text { (\%) }\end{array}$} & \multirow{2}{*}{$\begin{array}{c}\text { All } \\
\text { patients } \\
(\mathrm{N}=443)\end{array}$} & \multirow{2}{*}{$\begin{array}{c}\text { The conventional } \\
\text { CPR period } \\
(2019.01-2020.03) \\
(\mathrm{N}=267)\end{array}$} & \multirow{2}{*}{$\begin{array}{l}\text { The COVID-19 safety } \\
\text { protocol period } \\
(2020.04-2020.12) \\
(N=176)\end{array}$} & \multicolumn{2}{|c|}{ The COVID-19 safety protocol } \\
\hline & & & & $\begin{array}{c}\text { Crude OR } \\
{[95 \% \mathrm{CI}]}\end{array}$ & $\begin{array}{c}\text { Adjusted OR } \\
{[95 \% \mathrm{CI}]}\end{array}$ \\
\hline $\begin{array}{l}\text { Hospitalization } \\
\text { survival }\end{array}$ & $\begin{array}{c}75 \\
(16.9 \%)\end{array}$ & $50(18.7 \%)$ & $25(14.2 \%)$ & $\begin{array}{c}0.72[0.43- \\
1.21]\end{array}$ & $\begin{array}{c}0.61[0.32- \\
1.18]\end{array}$ \\
\hline $\begin{array}{l}\text { ROSC after hospital } \\
\text { admission }\end{array}$ & $\begin{array}{c}152 \\
(34.3 \%)\end{array}$ & $89(33.3 \%)$ & $63(35.8 \%)$ & $\begin{array}{c}1.12[0.75- \\
1.66]\end{array}$ & $\begin{array}{c}1.11[0.69- \\
1.79]\end{array}$ \\
\hline $\begin{array}{l}\text { 1-month survival } \\
\text { after OHCA }\end{array}$ & $\begin{array}{c}23 \\
(5.2 \%)\end{array}$ & $14(5.2 \%)$ & $9(5.1 \%)$ & $\begin{array}{l}0.97[0.41- \\
2.30]\end{array}$ & $\begin{array}{c}1.14[0.37- \\
3.50]\end{array}$ \\
\hline
\end{tabular}

Values are number (percentage). Confounding variables included resuscitation under the COVID-19 safety protocol, age, presence of witness, presence of bystander CPR (cardiopulmonary resuscitation), initial cardiac rhythm at the scene, call-hospital interval, and the first documented cardiac rhythm at hospital arrival. CI, confidence interval; COVID-19, coronavirus 2019; OHCA, out-of-hospital cardiac arrest; OR, odds ratio; ROSC, return of spontaneous circulation 
Figures

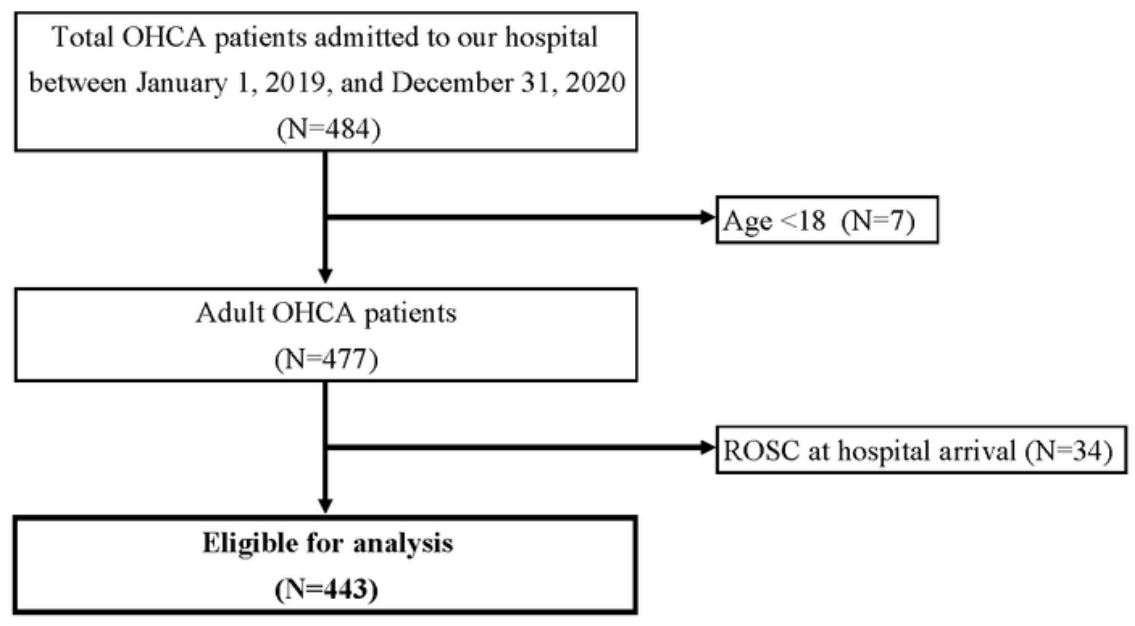

\section{Figure 1}

Flowchart of the study population.

\section{Supplementary Files}

This is a list of supplementary files associated with this preprint. Click to download.

- Additionalfilever.12021.03.01.docx 